\title{
Incoherentism and the Sorites Paradox
}

\author{
Matti Eklund \\ Uppsala University
}

Forthcoming in Sergi Oms \& Elia Zardini (eds.), The Sorites Paradox, Cambridge University Press.

\section{Introduction}

Consider the sorites paradox, and other philosophical paradoxes such as the liar paradox. The standard way of approaching them is by appeal to the semantic values by which I mean something at the level of reference, broadly construed - of the key expressions employed in stating the paradoxes. A central plank in such an approach is often a logic of vagueness or a formal theory of truth. The friend of the approach then tries to explain the phenomena at hand by appeal to these semantic values. Call an approach like this a reference-based approach to a paradox. The simplest kind of reference-based approach seeks to resolve the paradox by appeal to the nature of those things that the key expressions represent. For example, one might seek to resolve the sorites paradox, which has its source in vagueness, by saying that vague predicates stand for vague properties, and then give a theory of the nature of these vague properties. Call such an approach metaphysical. Not all reference-based approaches to paradoxes need be metaphysical. One prominent approach to vagueness and the sorites paradox is supervaluationism. ${ }^{1}$ The supervaluationist does not hold that the semantic values of vague expressions are in and of themselves vague. Instead she says that vague expressions are semantically indeterminate, and these expressions refer to different things under different possible precisifications. Supervaluationism does not seek to explain the sorites paradox by appeal to the nature of the individual referents of vague expressions, so it is not a metaphysical approach. But supervaluationism is still a reference-based approach, seeking to explain vagueness by appeal to facts about the reference of vague expressions; specifically, by appeal to the fact that these expressions have a multitude of candidate referents.

Of course, there are alternatives to reference-based approaches to paradoxes. One could at least in principle simply deny that vague language, or truth talk, is factual, and attempt to use this as the basis for a response. ${ }^{2}$ Perhaps the friend of this approach could say that our failure to resolve the sorites paradox is somehow due to a mistaken assumption to the effect that the vague language is factual. Or one could say that in addition to their semantic values, the relevant expressions have associated with them rules of use, not relating in any immediate way to contribution to truth-conditions, and

${ }^{1}$ The classic reference is Fine (1975). See also Keefe (2000).

${ }^{2}$ See MacFarlane (2016) for a recent approach of this kind. 
the paradox in question is explained by appeal to features of these rules of use.

The incoherentist view on the sorites paradox which I favor is another alternative to reference-based approaches. This view says, roughly, that the paradoxes arise because of an inconsistency in meaning-constitutive rules. These rules do not operate alongside and independently of truth-conditions but also help determine the truth-conditions of sentences containing vague expressions. Since the rules are inconsistent, the truth-conditions cannot be such as to actually validate the rules. This, the thought is, explains why it is so hard to come up with a satisfactory account of the truth-conditions of vague sentences. This incoherentist view does not break as radically with the tradition of explaining paradox by appeal to truth-conditions as the two suggestions previously mentioned do, for the rules she appeals to are held to be relevant to the determination of truth conditions. However, comparing these other alternatives is still helpful when it comes to illustrating the sort of strategy the incoherentist employs.

In what follows I will explain the incoherentist view I favor, present what I see as main arguments for such a view, discuss some purported objections, and compare other incoherentist views in the literature. ${ }^{3}$ While some of what I say explains and summarizing what is in the existing literature, primarily the discussion of objections contains much that is novel.

\section{Incoherentism}

When briefly characterizing incoherentism just above, I spoke of meaning-constitutive rules. Perhaps I should have used scare quotes around 'rule' to distance myself from this way of speaking. Lots of things can be, and have been, meant by talk of semantic rules, and I would want to distance myself from most of them. Even so, my own appeal to 'rules' will still be controversial.

What I am supposing - later I will return to this supposition, but for now just bear with me - is that to employ an expression with a given meaning is to take on certain commitments, or to have certain kinds of dispositions to use the expression in a given way. Some of these commitments or dispositions may fail to be consistent with each other. My favored version of the view focuses on dispositions instead of commitments, and in what follows I will focus on my favored version of the view.

\footnotetext{
3 I have defended incoherentism about vagueness in, e.g., my (2002) and (2005). Other incoherentists include Dummett (1975) and Horgan (1994). Nihilism, the view according to which vague predicates fail to be true of anything, defended by e.g. Peter Unger (1979a, 1979b, 1979c, 1980), Wheeler (e.g. 1975, 1979), Heller (1990), Ludwig and Ray (2002), and Braun and Sider (2007) is often mentioned alongside incoherentist views. (Williamson (1994), ch. 6, mentions Dummett as a nihilist.) But nihilism is importantly different from incoherentism. Saying that an expression is governed by inconsistent rules is one thing; saying that it is empty is another. The former may be held to entail the latter (though that is not my view - see below); it is not in the least plausible that the latter entails the former. I will return to nihilism (as defended by Unger) below.
} 
We may then say that a given language is inconsistent exactly if competence with all elements of that language involves dispositions to accept things that are inconsistent with each other. Talk of language being inconsistent hardly has a pretheoretically clear meaning. I am using the locution partly stipulatively.

The account of competence just sketched does not by itself say anything about the semantic values of the expressions of an inconsistent language. One perhaps natural suggestion would be that when the rules governing an expression are inconsistent, then the expression simply lacks a semantic value (or it has a trivial semantic value: for example, a predicate would be empty). I see two problems with this. First, this seems implausibly radical: it yields that nothing is old, or red, or a mountain, etc. Anyone who embraces this radical idea is apt to want to draw a distinction between assertible and non-assertible sentences of, for example, the form '___ is old', and so mitigate the problem by saying that those of these sentences we would take to be true are at least assertible. However, deciding under what conditions sentences are assertible presents much the same problems as deciding under what conditions they are true, and supposing one can come up with a satisfactory theory of assertibility, why not simply use that also as a theory of truth? Second, a different problem is that the incoherence is holistic. It is not just the rules for one expression that lead to incoherence. The rules governing vague expressions lead to problems only jointly with assumptions about logic. 4 If the incoherence is holistic, exactly which expressions should then be taken to be empty?

Instead I prefer a view that allows vague expressions to have non-trivial semantic values despite the fact that vagueness is inconsistent in the way indicated. Here is one way of allowing for this. Consider the theory of reference-determination in the spirit of Lewis. ${ }^{5}$ Following Carnap, Lewis held theoretical terms to be somehow implicitly defined by the theories in which they occur. In the simplest case, the referent of a theoretical term is whatever makes true the sentences of the theory wherein the term occurs. But we certainly want to say that theoretical terms occurring in false theories can refer, so we can't always say exactly that. What Lewis does is to allow that the term refers to what comes closest, and close enough, to making true those sentences of the theory. Closeness is here not just a matter of what, quantitatively, makes true the most sentences. Overall best fit is what is at issue; qualitative considerations enter in as well. And if it turned out that a given theory was inconsistent, the same would apply. Inconsistency is not relevantly different from mere falsity. It is presumably obvious where I am going with this: the relationship between the semantic values of vague expressions and the rules, in the sense gestured at, which govern them is, I suggest, like that between the semantic value of a theoretical term introduced by an inconsistent

4 See e.g. Zardini (2008).

5 See e.g. Lewis (1970), (1972), and (1997). 
theory and the sentences of that theory.

It is reasonable to speculate that it is indeterminate just what the semantic values of vague expressions are: it is indeterminate what assignment comes closest to making true the principles that function as meaning-constitutive rules. But vague expressions have non-trivial semantic values; it is only that it is indeterminate what these non-trivial semantic values are.

These general remarks are consistent with many different specific views regarding what the semantic values of the relevant expressions are. The main point for present purposes is that a language being inconsistent need not be bound up with the relevant expressions having trivial semantic values, or lack semantic values altogether. It is possible, of course, to think that what has just been sketched is only one factor in reference-determination, and other considerations enter in as well. One might even hold that the principles it is part of competence to be disposed to accept do not contribute in any direct way to reference determination: instead some kind of causal theory of reference is correct.

If languages can be inconsistent in the way characterized, one can attempt to diagnose some philosophical paradoxes as arising from inconsistency of our language. The form of the diagnosis is that among the assumptions that lead to absurdity through the paradoxical reasoning are some that are untrue but underwritten by competence.

In the case of vagueness, the present topic, a natural way to spell out the incoherentist idea would be to say that tolerance principles are meaning-constitutive for vague predicates, where a tolerance principle for a predicate $\mathrm{F}$ is:

Tolerance: Some small enough difference in F's parameter of application never matters to F's applicability, even while some large enough difference sometimes does.

It is tolerance principles that underwrite the sorites paradox. Take a specific version of the paradox:

(1) Someone who is $1400 \mathrm{~mm}$ tall is not tall.

(2) For all $\mathrm{n}$, if someone who is $\mathrm{n} \mathrm{mm}$ tall is not tall, then someone who is $\mathrm{n}+1 \mathrm{~mm}$

tall is not tall.

(C) So, someone who is $2900 \mathrm{~mm}$ tall is not tall.

The 'some small enough difference never matters' part underwrites the major premise, (2); the 'some large enough difference sometimes matters' underwrites both that there will be an intuitively acceptable premise like (1) and an intuitively unacceptable 
conclusion like (C).

My view is that not only is vagueness a source of inconsistency in language of the kind outlined, but also that this is crucial to understanding vagueness and the sorites paradox. I will defend this claim in the next section. One could in principle agree that vagueness is a source of inconsistency in the way described, but hold that this is not very interesting - that the key to obtaining a theoretical understanding of vagueness and the sorites lie elsewhere. Consider in this connection what I said above about semantic value. The dispositions constitutive of competence are dispositions to accept incoherent things, but help determine consistent, non-trivial truth-conditions. This means that the incoherentist view I defend is in principle compatible with any standard view on the truth-conditions of vague sentences found in the literature, and does not immediately provide support for one of these views over others. Philosophers of a certain bent of mind will then ask: what is the point? Doesn't the incoherentist suggestion leave everything as it is? This is a possible and understandable reaction, but it betrays commitment to a reference-based approach to the paradox. For what it amounts to is that suggestions that do not have any immediate implications regarding reference do not have any important implications as far as the paradox is concerned.

\section{Arguments for incoherentism}

If the incoherentist view is correct, that nicely explains a number of phenomena related to vagueness and the sorites. In this section I will go through these things.

Recalcitrance. The hypothesis explains the seeming irresolubility of the sorites paradox: why every proposed solution seems intuitively unsatisfactory. A natural way to see the literature is this. Proposed semantics for vagueness leave the extensions of vague predicates with unintuitively sharp boundaries; new semantics are proposed, meant to get around this; those semantics are argued not to avoid the problem; and so on. A seeming problem with a fully classical and bivalent theory is that an arbitrarily small difference in the relevant dimension given this theory implausibly makes for a difference between it being true that something is $\mathrm{F}$ and false that something is $\mathrm{F}$, for $\mathrm{F}$ a vague predicate. If in response to this problem a many-valued or fuzzy theory is proposed, a version of the original problem remains. There are still unwanted boundaries, this time between, e.g., the true and the neither-true-nor-false, or the perfectly true and the less than perfectly true. A seeming lesson of the philosophical discussion of the sorites paradox is that this generalizes. Any otherwise acceptable assignment of semantic values to expressions of our language yields unwanted boundaries. Incoherentism does not get around this. Nor is it meant to. What it does yield is that no assignment of truth-conditions to vague sentences will fully satisfy the meanings of vague expressions. It then predicts the phenomenon of recalcitrance. The incoherentist can say that one cannot expect there to be an otherwise acceptable assignment of truth-conditions to vague sentences that avoids these unintuitively sharp boundaries. All 
one can hope for is that some assignments come close enough to respecting the meaning of vague expressions that these expressions have semantic values. Needless to say, friends of specific accounts of vagueness found in the literature will think they have their own ways of getting around the problem, and to properly make an argument from recalcitrance one would need to discuss individual proposals separately.

Flip-flopping. The issue of recalcitrance has to do with what happens when we try to theorize about vagueness. Focus now not on theorizing about vagueness but on the actual use of vague expressions. Arguably, one distinctive feature of what happens in sorites cases is that speakers are apt to flip flop. An item that is classified one way at first can be classified a different way later and the speaker never comes to a stable verdict. This phenomenon underlies the appeal of contextualist account of vagueness. ${ }^{6}$ But it is also neatly explained by appeal to the idea that vague expressions are governed by inconsistent 'rules'. Compare: You work in a paint shop. You're being told that paradigmatic red paint goes in the left corner and paradigmatic yellow paint goes in the right corner. For any two jars of paint, if the paint in the jars is sufficiently alike they should go in the same corner. If the paint shop is sufficiently well stocked, the jars of paint constitute a sorites series, and there is no way you can obey these instructions. Assuming you don't notice the inconsistency in the instructions, you will vacillate, in a way familiar from the vagueness literature. Having classified a jar of paint one way and put it in the right corner you can notice its similarity to jars of paint put in the other corner and so revise your verdict on that, but having done so, you will think other jars of paint put in the right corner should be reclassified. But then... Of course, you will soon realize the futility of your undertaking; but the same applies in the case of vague expressions. Any classification is bound to fail to respect a meaning-constitutive principle governing the expression. That does not mean that vague predicates are empty any more than the paint store employee succeeds in her task by not dividing up the jars at all.

The nature of vagueness. 7 What is it for an expression, for example a predicate, to be vague? Here is one kind of suggestion found in the literature:

(BORDERLINE) For a predicate to be vague is for it to have possible borderline cases.

But what is a borderline case? Do we have an independent handle on what borderline cases, in the relevant sense, are? To elaborate: If a predicate $\mathrm{F}$ has borderline cases if and only if some sentences of the form " is F' are neither true nor false, then there are phenomena other than vagueness which give rise to borderline cases. For (BORDERLINE) to provide an acceptable account of the nature of vagueness

${ }^{6}$ See e.g. Fara (2000), Kamp (1981), Raffman (2014), Shapiro (2006), and Soames (1999).

7 This theme is developed in Eklund (2005). 
'borderline case' must mean something more specific. But here the worry arises that to elucidate to intended sense of 'borderline case' one must speak of lack of truth-value due to vagueness, and then we no longer have a very informative characterization of vagueness.

A different suggestion is:

(SORITES-SUSCEPTIBILITY) For a predicate to be vague is for it to be soritessusceptible.

But what is it for a predicate to be sorites-susceptible? For it to be such as to make some sorites reasoning sound? No, for the sorites reasoning is not sound. Is it then for it to make some sorites reasoning seductive - that it be such that we are actually disposed to be taken in by the reasoning? But that proposal yields that vagueness is just a psychological phenomenon. Suppose we stopped having the disposition to be taken in by sorites reasoning using 'old' - perhaps because of there being greater awareness of the problems with such reasoning. The proposal at issue does not immediately have the consequence that 'old' would change its meaning; but it does have the consequence that 'old' would cease to be vague. I think a reasonable proposal regarding vagueness makes vagueness a matter of meaning, in such a way that a vague expression cannot cease to be vague without change in meaning.

However, appeal to (SORITES-SUSCEPTIBILITY) does work if soritessusceptibility is written into the meanings of vague predicates in the way outlined in the characterization of the incoherentist view. Then the vagueness of an expression is not just a matter of whether we happen to be taken in by some associated sorites reasoning. It is a matter of something that is constitutive of the meaning of the predicate.

Vagueness and semantic indeterminacy. It is generally agreed that vagueness in some way gives rise to or is associated with semantic indeterminacy. Note the gives rise to or is associated with, rather than is identical with. It should be uncontroversial that there are sources of semantic indeterminacy other than vagueness, for example partial definition, and the indeterminacy phenomenon Hartry Field (1973) discusses in connection with Newtonian 'mass' 8 But even if vagueness is not identical to semantic indeterminacy one can wish for an account of how vagueness is connected to semantic indeterminacy.

Standard models for what the semantic indeterminacy associated with

\footnotetext{
8 What Field discusses is that given what we know today, there is no quantity which exactly satisfies the conception associated with mass in Newtonian physics. Instead there are two quantities - relativistic mass and rest mass - that both are best candidates. Field thinks 'mass' as used by Newtonians is semantically indeterminate as between relativistic mass and rest mass.
} 
vagueness would be like are unsatisfactory, connected to how theories of the sorites paradox generally are unsatisfactory. Saying that a vague predicate carves up the domain into three classes, those entities it is true of, those it is false of, and those such that it is indeterminate whether it applies, is unsatisfactory as it gives rise to unintuitive sharp boundaries.

Some theorists, those who appeal to metaphysical vagueness and those inclined to favor epistemicist accounts, may of course deny any connection between vagueness and semantic indeterminacy, and they can appeal to the problems in making good sense of the indeterminacy in question as part of their case for the denial. 9 However, I believe that the incoherentist view can serve as a basis for a nice account of the relation between vagueness and semantic indeterminacy. ${ }^{10}$

Distinguish between first-level indeterminacy and second-level indeterminacy:

A sentence is first-level indeterminate if it (due to indeterminacy) lacks classical truth value under some acceptable assignment of semantic values.

A sentence is second-level indeterminate if (due to indeterminacy) it has different truth values under different acceptable assignments of semantic values. ${ }^{11}$

An acceptable assignment of semantic values is here a best possible account of semantic values. An acceptable assignment in this sense is not the same thing as a precisification, as 'precisification' is used in the discussion of supervaluationism about vagueness. ${ }^{12}$ The way traditional supervaluationists think about it, vagueness gives rise to an expression's having a multitude of precisifications, corresponding to the ways of making the expression precise, compatibly with the meaning it is currently endowed with. Each precisification is classical. Moreover, a sentence is true iff it is true under all precisifications, false iff false under all precisifications, and neither true nor false iff it has different truth-values under different precisifications. Supervaluationism is only a framework for modeling first-level indeterminacy. The correct assignment of a semantic value to a sentence that receives different truth-values under different precisifications is neither true nor false.

\footnotetext{
9 However, arguably the unwanted boundaries problem arises also for the metaphysical indeterminacy theorist.

${ }^{10}$ See especially Eklund (2010b).

${ }^{11}$ The reason for the added "due to indeterminacy" in the first definition is that there may be other reasons for lack of classical truth value besides indeterminacy, for example presupposition failure. I know of no corresponding need for a similar clause in the second definition, but it does not hurt to include it. Because of the occurrences of this clause, the characterization of first- and second-level indeterminacy is not a reductive characterization of what indeterminacy amounts to.

${ }^{12}$ See again Fine (1975).
} 
Note that there can be second-level indeterminacy without such first-level indeterminacy: it can be that some sentences have different truth-values under different acceptable assignments but under no assignment does a sentence lack a determinate classical truth value.

The concern about standard accounts of the semantic indeterminacy associated with vagueness is that saying that vague expressions carve up their domains into more than two classes fails to get around the problem of unwanted boundaries. This is a strike against postulating that vagueness is bound up with first-level indeterminacy. (An assignment of semantic values to vague expressions given which there is first-level indeterminacy does not do appreciably better in accommodating the intuitive picture associated with vagueness that does an assignment of semantic values to vague expressions given which there is no such indeterminacy.) But it is reasonable to speculate that given an incoherentist view on vagueness, vagueness is regularly associated with second-level indeterminacy: if there is no way to satisfy the constraints imposed on truth-conditions by what is constitutive of meaning, why suppose there will still in general be a unique best assignment of truth conditions? ${ }^{13}$ One can then accommodate the sense that vagueness is somehow associated with semantic indeterminacy by saying that it is associated with second-level indeterminacy. If one likes, one can also say that it is also associated with first-level indeterminacy, but there is no longer the same theoretical need to say this. One has accounted for the connection to indeterminacy already by appeal to the tie to second-level indeterminacy. ${ }^{14}$ Of course, it is intuitively repugnant also to think that vagueness gives rise to there being a tripartite distinction between what is true under all acceptable assignments, what is true under some but not all acceptable assignments, and what is true under no acceptable assignments. But this intuitive repugnancy, although real, is not very pressing: for there is no reason to think our competence with vague expressions should be a reliable source regarding the structure of what acceptable assignments there are.

Returning to the question of indeterminacy and the problem of unwanted boundaries, here is the relevant point. Unwanted boundaries are in some way a flaw in a given assignment, since an assignment purports to be a correct representation of the semantic values of the expressions of the language, and the counterintuitiveness is a strike against it succeeding in this. But incoherentism says that we are stuck with unwanted boundaries. Any assignment will be flawed in the sense of not fully respecting the meaning-constitutive principles for vague expressions.

\footnotetext{
${ }^{13}$ This is not to say that incoherentism is the only view on vagueness that could have this consequence.

${ }^{14}$ In the literature, there are also general concerns about what semantic indeterminacy is supposed to be - see Merricks (2001) and Taylor and Burgess (2015). The suggestion mentioned does not immediately help with those concerns.
} 
I say that vagueness is associated with second-level indeterminacy, but I would not want to commit to the claim that every vague expression has to be associated with such indeterminacy. Suppose for example that reference magnetism is real: that some things expressions might mean are intrinsically more eligible to be meant (more 'reference-magnetic'). ${ }^{15}$ Then it can be that for some vague expressions there is a unique best assignment of a semantic value: that is the reference-magnetic semantic value in the vicinity, so to speak. Among what otherwise would have been a multitude of acceptable assignments, there is one that has the expression stand for something reference-magnetic. I would say that in this case, the expression is vague but not semantically indeterminate. That seems to me to be the right result. But I wouldn't rest any argumentative weight on it, but regard these cases as spoils to the victor. Alternatively, one could distinguish two different kinds of vagueness, vagueness with respect to sense and vagueness with respect to reference, and say that an expression has the latter feature only if it has different semantic values under different assignments. ${ }^{16}$

\section{Objections regarding competence}

Turning to objections to the incoherentist view proposed, let me start by, in this section, considering the most obvious one: while incoherentism might be nice if it works, it requires an implausible view on meaning and competence. The underlying view is what I will call an SCR-view, where the SCR stands for selective conceptual role. It is a conceptual role view, for it associates an expression with a particular role in a speaker's cognitive economy - that characterized by the dispositions vis-à-vis the meaningconstitutive-principles - and says competence with an expression involves associating it with the right role. It is selective, for as opposed to holistic views, it says that only some

15 The label reference magnetism is from Hodes (1984). The idea as it has come to be discussed derives from Lewis $(1983,1984)$, although there is a discussion in the literature regarding what exactly Lewis' views on this were - see e.g. Schwarz (2014).

${ }^{16}$ There is a connection between the themes brought up in the present section and epistemicism. If "epistemicism" is understood simply as the view that vagueness is compatible with both bivalence and classical logic, then the above suggests a defense of epistemicism different from those prominent in recent discussions. The idea is that each acceptable assignment is fully bivalent (at least as far as vagueness is concerned) and the only way the indeterminacy comes in, is through there being different acceptable assignments. Since bivalence holds under each acceptable assignment, bivalence holds simpliciter. Already Campbell (1974), an early defense of some sort of epistemicism, brought up a version of this idea, under the label "semantic uncertainty". (The label "uncertainty" is not ideal: it suggests that the phenomenon is merely epistemic.) Compare too Nick Smith (2008) on 'plurivaluationism'. Smith essentially defends a fuzzy view on vagueness but with the twist that he adds that there is (what I call) second-level indeterminacy: different assignments ascribing different (fuzzy) truth-values to sentences are all acceptable.

One main argument for epistemicism is to the effect that there is no avoiding unacceptable sharp boundaries anyway. But despite the undeniable strength of those arguments, it is hard to swallow the consequence that something about our use of expressions determine a unique best assignment of semantic values. The current proposal accepts those epistemicist arguments while avoiding this consequence. 
of the representations involving the expression that one accepts or is disposed to accept are constitutive of what meaning one uses the expression with.

There are two different perspectives from which one can attack the underlying view on meaning and competence. One can accept an SCR-view on competence but hold that for general reasons what competence disposes one to accept can never be incoherent in the way that incoherentism postulates, or one can reject an SCR-view in favor of something different entirely, for example the view that to be competent with an expression just amounts to having some capacities or other in virtue of which one uses it with the customary reference. Let me start by considering the view that although an SCR-view is acceptable, languages cannot be inconsistent in the way discussed.

A problem for an SCR-view that aims to leave room for meanings to be inconsistent is that anyone who realizes that some set of principles lead to inconsistency - for example a philosopher studying the sorites paradox - will plausibly thereby be disinclined to accept all of these principles. And we don't want to say that this philosopher thereby loses her semantic competence.

However, this problem arises for any SCR-view given when the cognitive relation between a competent thinker and the meaning-constitutive principles is something like belief. But already independent considerations show that such an identification is too simple-minded. If anything has the status of a meaning-constitutive principle, the sentences expressing basic logical laws surely do. But for pretty much any basic logical law, one can find logicians and philosophers rejecting the sentences expressing them; and since these logicians and philosophers are (the argument goes) unquestionably competent, these sentences cannot be meaning-constitutive. ${ }^{17}$ Hence, the argument runs, it is somewhat trickier than might have been expected to identify the cognitive relation in question. But then simple-minded suggestions regarding the nature of the cognitive relation anyway do not work.

What might the cognitive relation plausibly be? Any discussion here will have to be brief. But one suggestion is that it is something like taking it to be the default position that [...]. (Implicitly, of course. The claim is not that speakers actually think if the expressions they use in these terms.) Strikingly, counterexamples to taking the cognitive relation to be something like (dispositions to) belief or acceptance always involve someone having a special reason to deny the supposed analyticity. Timothy Williamson has prominently called attention to Vann McGee's rejection of modus ponens when criticizing the notion of epistemic analyticity, and he has also called attention to how speakers can for specific reasons reject instances of the schema 'every $\mathrm{F}$ is an F'. But strikingly, the examples involve thinkers armed with specific reasons to reject the epistemic analyticities. If one imagines someone rejecting modus ponens or

${ }^{17}$ See Williamson, e.g. (2007), for discussion. 
instances of 'every F is an F' without special reason to do so, it becomes considerably more doubtful that this thinker - social externalism ${ }^{18}$ aside - uses these terms with their standard meanings. ${ }^{19}$ Another suggestion is to turn to normative characterizations, and speak of what speakers are entitled to accept instead of what they actually believe or are disposed to believe. ${ }^{20}$

If the meaning-inconsistency view couldn't be combined with a plausible view on reference-determination - if, say, the meaning-inconsistency view had the consequence that the semantic values of vague expressions are undefined or empty then there could be reason for a friend of an SCR-view to reject the possibility of a meaning-inconsistency view at least for philosophically significant cases. But I argued above that the Lewisian picture of reference-determination straightforwardly allows that vague predicates have non-empty extensions, even if inconsistent in the way outlined.

Next, turn to Wright's important (1975) discussion of incoherentism about vagueness. (Wright's immediate target was Dummett (1975), which I will get to later.) Wright thinks meaning-consitutive principles cannot be inconsistent. One central point he makes is that meaning-constitutive rules cannot be inconsistent while sufficing, by themselves, to confer meaning upon an expression: since any use of the expression can be made to fit with the meaning-constitutive rules, these rules do not rule out anything. ${ }^{21}$ He says for instance that ' $[\mathrm{t}]$ he rules of [a game supposed to be governed by inconsistent rules] do not provide an account of how the game is played, for it is possible that someone might grasp them yet be unable to participate'.22

However, distinguish between the claim that a full account of an expression's meaning (or of how a game is played) can be provided by a mere list of inconsistent rules (or as I would prefer to put it when speaking in my own voice, meaningconstitutive principles), and the claim that some rules governing an expression are individually or jointly inconsistent. What Wright casts doubt on is the former claim, but incoherentism is only committed to the latter. Surely the view that meaningconstitutive principles can be inconsistent does not carry with it a commitment to the view that our competence can be exhaustively described by appeal to a mere list of these inconsistent rules: matters like how the rules are to be weighted are also relevant.

Some theorists want nothing to do with SCR-views, the possibility of

\footnotetext{
${ }^{18}$ Social externalism is, roughly, the view that what an expression means as used by a speaker can be determined in part by the speaker's social environment.

19 See Williamson (2007), and, for discussion, Eklund (2007) and (2010a). Williamson uses these examples to criticize SCR-views; what I hold is that the examples only puts pressure on how SCR-views are best construed. Wright (2004), p. 169f, gestures toward the sort of idea I am describing here.

${ }^{20}$ See Scharp (2013), ch. 2.

${ }^{21}$ See Wright (1975), pp. $361 \mathrm{ff}$.

22 Wright (1975), p. 362.
} 
incoherentism aside. Let me turn to this. Prominent among such theorists are those who hold that all there is to the semantics of an expression is its reference, plus aspects that do not directly relate to truth-conditions, such as conventional implicature - they eschew the notion of meaning-constitutive principles entirely. Call these theorists referentialists. ${ }^{23}$ It would obviously take us too far afield to assess fully generally whether a referentialist view or an SCR-view is preferable. But some things specifically pertaining to the case of vagueness are worth bringing up.

One thing I would press against a referentialist is that she will have a hard time accommodating vagueness as genuinely a feature of the meaning of an expression. If the meaning of an expression just consists in its reference, then the only way an expression can be vague is if its referent is vague, or maybe if, along the lines of supervaluationism, it has different candidate referents - but these resources are too meager to capture vagueness, for there can be non-vague expressions that behave referentially in the same way that vague expressions do. Suppose for example that a simple fuzzy theory is the correct theory of vagueness, and suppose that someone is tall* to degree 1 iff that person is at least $1900 \mathrm{~mm}$, tall* to degree $\mathrm{o}$ iff that person is $1800 \mathrm{~mm}$ or less, and tall* to degree $d$ if in between, where $d=$ (the person's height in $\mathrm{mm}-1800$ ) / 100. 'Tall ${ }^{*}$ ' is hardly vague, despite having a fuzzy semantics. There is no reason why it would invite a sorites paradox; the principle that a tiny difference in height makes no difference to tall*ness lacks plausibility. Or compare a simple version of supervaluationism, where what marks an expression as vague simply is its having different possible precisifications. Compare then a predicate 'nice', stipulated to behave as follows: a number is nice if $<13$; not nice if $>15 .{ }^{24}$ One might add that for any $n$, if $n$ is nice then $n$ 1 is likewise nice. 'Nice' then has different precisifications, since nothing has been said about the classification of 13, 14 and 15. 'Nice' behaves referentially like vague predicates behave according to this simple version of supervaluationism, but it is not vague. Now, a simple fuzzy theory and simple supervaluationism are, precisely, simple theories. For all I have actually argued, more sophisticated theories could succeed in characterizing vagueness referentially. That would have to be judged on a case by case basis. But I believe the strategy illustrated by these simple cases generalizes. Importantly, the argument is not to the effect that what the theories say about the referential properties of vague expressions is mistaken but only that it does not suffice to characterize vagueness.

Some theorists believe in metaphysical vagueness. They may think that vague singular terms refer to vague entities, and vague predicates ascribe vague properties; and that the vagueness of the expressions is due to the vagueness in the entities that they refer to and ascribe. If these theorists are right, then of course something about the

${ }^{23}$ The label is from Williamson (2009).

${ }^{24}$ Compare Fine (1975), p. 266. 
reference of vague expressions separates them from non-vague expressions: all and only vague expressions refer to vague entities. The referentialist can say that this distinguishes the reference of vague expressions from the reference of other expressions.

Elsewhere, I have given separate arguments against metaphysical vagueness. ${ }^{25}$ But there is a point to be made even if metaphysical vagueness does obtain. Let the truth value-contribution of an expression be the complete set of facts regarding what it contributes to the truth-values of sentences in which it is used. Using this notion, the point of my above argument is that the truth value-contribution of a vague expression can be the same as the truth value-contribution of a non-vague expression, and this point stands even given supposing there is metaphysical vagueness. And this by itself means that theorizing about the reference of vague expressions is unlikely to hold the key to the puzzles surrounding vagueness.

What I have been talking about is just an instance of a more general problem for the referentialist way of thinking about meaning. Consider normative language. Some expressions ('right', 'wrong', etc.) seem to be normative as a matter of their meanings. A referentialist would have to say that any such expression would have to classify as such in virtue of what it refers to. But this is problematic: normative and non-normative expressions can corefer. A quick argument to that effect is that one could easily introduce a non-normative expression with the same reference as a normative expression (consider for example 'let "flurg" refer to whatever the third word uttered by someone over six feet tall in the year 2073 refers to', and suppose that third word is 'right'). ${ }^{26}$

One suggestion for a would-be referentialist who happily lives with the consequence that vagueness and evaluativeness cannot be semantic features is that she might take these features to be metasemantic rather than semantic: these are not features of the meaning of an expression but have to do with how the reference of the expression is determined. In the case of vagueness, she can say that the vagueness of an expression has to do with how its reference is determined. She can then defend a slimmed down version of what the incoherentist holds: the language users' acceptance of tolerance principles help determine the reference of vague expressions.

\section{Further objections}

I have discussed at some length objections to incoherentism that focus on the underlying conception of meaning and competence. Let me now turn to some other objections.

Charity. One possible objection is that even if inconsistent languages like those

\footnotetext{
25 See Eklund (2013).

${ }^{26}$ I discuss this in ch. 4 of Eklund (forthcoming).
} 
described are possible, charity militates against the hypothesis that we speak such an inconsistent language. Even though it is a theoretical possibility that we speak such a language, other interpretive possibilities are vastly preferable. But there's a question of how charity is supposed to come into it. Charity would enter into it most obviously if the incoherentist view but not opposing views ascribed incoherent beliefs to us. But such a claim would be doubly mistaken. First, and most importantly, on any reasonable view, we are prone to incoherent beliefs when it comes to vagueness. That is why the sorites paradox arises. Second, a plausible version of incoherentism speaks not of what competence demands that we believe but instead of, for example, what competence disposes us to accept. This means that appeal to charity has even less purchase.

Note too that in principle it can be overall more reasonable for a thinker to employ quick but dirty rules - rules that are easy to employ but which may lead the thinker astray in some special cases - than for her to employ corresponding considerably more complex rules guaranteed never to lead her astray but considerably less economical to employ. ${ }^{27}$ All else equal, an interpreter deciding which of these sets of rules to interpret a thinker as employing may well be led by some charity-like principle positively to prefer interpret the thinker as employing the quick but dirty rules.

The consideration just presented works best if one understands 'rules' in the context as an algorithm the thinker's cognitive system employs. This goes beyond how 'rules' has been glossed earlier in the discussion and one must hence be careful. There still is a general lesson in the vicinity.

Tolerance without incoherence. The last few years have seen an increase in interest in accepting tolerance principles at face value but making such revisions in logic - specifically in what structural rules are accepted - that this does not lead to absurdity. ${ }^{28}$ This is one way in which the theoretical landscape has changed since when I first defended the incoherentist view here outlined. Any theorist who can accept tolerance principles at face value can adopt the account of the nature of vagueness that I presented. So the existence of views like that described shows that the argument concerning the nature of vagueness and the argument from flip-flopping do not favor incoherentism over all possible competitors, even if these arguments are otherwise good as far as they go. I still think incoherentism is to be preferred over these nonincoherentist tolerance views. For one thing, I think incoherentism is uniquely well placed to capture the sense that there can be no fully adequate account of the truthconditions of vague sentences; and, relatedly, explain the phenomenon of recalcitrance. Of course, to make this point against those who favor non-incoherentist theories that respect tolerance it would have to be shown that these theories are indeed

${ }^{27}$ See e.g. Cherniak (1984).

${ }^{28}$ See e.g. Zardini (2008). 
unsatisfactorily counterintuitive. I will not attempt to do this here.

One way in which the rejection of structural rules differs from other proposed revisions of logic is in that whereas one can see logics that differ over the semantics of individual logical expressions as corresponding to different languages, structural rules are not tied to language in the same way. The reason this is relevant is the following. So long as we are dealing with descriptions of different possible languages one can say that the question of which possible language we speak is to be decided by a theory of interpretation. But if structural rules are not tied to language in the same way, things may look different. However, although structural rules may not strictly be part of language it can still obviously be that different thinkers, or different groups of thinkers, employ different structural rules. A theory of interpretation for a thinker or group of thinkers will then concern not only what language is employed by also such matters as what rules of reasoning are employed; and when it comes to the question of which interpretation is correct, it is a package deal.

Vagueness without tolerance. Much of the supposed attraction of the view stems from its supposed ability to satisfactorily answer the question of what the nature of vagueness is. In other words, it is due to the attraction of saying that tolerance principles are meaning-constitutive for vague expressions. But not all vague expressions are plausibly governed by tolerance principles. First, tolerance principles are formulated for predicates, but there are arguably vague expressions of other categories (consider, for example, vague quantifiers). Second, even focusing on the specific case of predicates, what about a predicate like 'has few children for an academic'? This seems to be a vague predicate, but because the associated sorites series would be so short, there is no associated compelling version of the sorites paradox. ${ }^{29}$

Complexity. By far the most serious objection to incoherentism, as far as I am concerned, is this. Vagueness would seem to be a ubiquitous feature of languages. But if the proposed incoherentist view on vagueness were correct, that would hardly be so. For why would it be a ubiquitous feature of languages to have meaning-constitutive principles of this rather peculiar kind for a large range of predicates? Other theories more readily account for the ubiquity of vagueness. On a theory on which vagueness is at bottom metaphysical, a language is vague simply because it contains expressions referring to the vague things in the world. On a supervaluationist theory, vagueness arises simply because of semantic indecision. Call this objection to incoherentism the objection from complexity.

One incoherentist response to this objection is to just insist upon the arguments in favor of the view, and say that these arguments yield that we must accept the view: maybe it is antecedently unikely that what the incoherentist postulates is a ubiquitous

${ }^{29}$ See Weatherson (2010) for this kind of challenge; and see Eklund (2006) for some discussion of it. 
feature of languages, but somehow or other it must be so. This response can be bolstered by closer consideration of the supposedly simpler theories that would avoid the objection from complexity.

An incoherentist can try to present the supervaluationist with a dilemma. A very simple form of supervaluationism will say that an expression's vagueness consists in the expression having multiple precisifications. This feature is likely to be ubiquitous. There will be vagueness thus conceived in all natural languages. But this simple supervaluationism will not do. Any partially defined expression will be classified as vague. If in response to this the supervaluationist complicates her theory, she will face the same sophistication problem as incoherentism does. Why think this complex phenomenon would be thus ubiquitous? The supervaluationist then faces a version of the problem of complexity.

A similar issue arises when it comes to metaphysical vagueness. On a simple view on metaphysical vagueness, vagueness is plausibly ubiquitous but the simple view is too simple to be satisfactory; given a more complex view the ubiquity is again hard to explain. An example of a simple view would be one where metaphysical vagueness simply gives rise to there being some sentences lacking classical truth-values. A more sophisticated view tries to get around the obvious problems faced by this simple account by adding complexity, but then a version of the problem of complexity arises.

\section{Related views}

In this last section let me consider some views in the literature similar to mine - those of Terence Horgan (1994), Peter Unger (1979a, 1979b, 1979c, 1980) and Michael Dummett (1975). Horgan and Dummett are plausibly classified as incoherentists, and Unger's view is similar in spirit. ${ }^{30}$

Horgan (1994) presents a kind of thesis-antithesis-synthesis argument where the thesis is that vagueness is impossible (because of leading to contradiction), the antithesis is that vagueness is possible (because clearly actual) - and the synthesis is

Vagueness in THE WORLD is impossible; vagueness in thought and in language is incoherent and yet is actual (and hence possible) anyway. ${ }^{31}$

The world itself cannot be vague, but our representations of it can be. It is only that vague language and thought is incoherent. This is of course completely consonant with the incoherentist view that I have stated.

Horgan is rather cavalier about the nature of the supposed incoherence in

\footnotetext{
${ }^{30}$ As discussed in fn3, Unger's view is not strictly an incoherentist view. It is, however, similar enough that comparing Unger's view is useful.

${ }^{31}$ Horgan (1994), p. 179.
} 
thought and language. He says 'For purposes of this paper I can leave it open how best to cash the general notion of logical incoherence.... Roughly, a concept is logically incoherent if someone who employs it correctly thereby becomes committed, at least implicitly, to accepting statements that jointly entail a contradiction'. ${ }^{22}$ He does not directly address the question of how the supposed incoherence affects the truthconditions of vague sentences, but instead spends some time arguing that the incoherence is insulated in such a way that it does not 'propagat[e] itself destructively through our thought and discourse'.33 That point is actually rather obvious, independently of the specifics of incoherentism: for somehow or other we can have inconsistent beliefs without that having seriously damaging consequences. It is not clear why having inconsistent meaning-constitutive beliefs (or dispositions thereto) would need to be any different in that regard.

Peter Unger (e.g. 1979a) holds that when $\mathrm{F}$ is a 'vague discriminative term' the following holds:

There is some relevant dimension of difference D such that (i) if something differs from an F minutely along $\mathrm{D}$, then it also is an F, and (ii) if something is an F, then there is some actual or possible differing substantially along D which is not an F.34

This yields via familiar sorites reasoning that there are no Fs. The assumption that something is $\mathrm{F}$ leads to contradiction. 35

Unger's reasoning leads to many radical conclusions. There are no heaps, no clouds, no persons, no one knows anything, etc. To my mind, Unger simply overlooks the sort of possibility that I explore in what I say about reference-determination. Even if Unger is completely right about what principles in some sense govern vague predicates, the semantic values of vague expressions can fail to render them true.

It is in principle possible, of course, to attend to the possibility I call attention to and still conclude with Unger that vague expressions are empty. One can have a theory of how meaning-constitutive principles constrain or determine semantic values that yields that incoherence always carries emptiness in its wake. Or one can, in principle, allow that expressions governed by incoherent meaning-constitutive principles are nonempty but hold that features specific to the case of vagueness make it the case that vague expressions are empty: for example, one could hold that tolerance is so central to the use of vague predicates that no assignment of semantic values that fails to respect

\footnotetext{
$3^{2}$ Horgan (1994), p. 180.

33 Horgan (1994), p. 180.

34 Unger (1979a). The exact formulation in the text is my own. Unger's own statements are found on p. 181 (rough version) and p. 182 (more careful general version).

35 Other relatively recent theorists who have held that vagueness implies emptiness or lack of truth include Wheeler (e.g. 1975, 1979), Heller (1990), Ludwig and Ray (2002), and Braun and Sider (2007).
} 
tolerance comes 'close enough'. But let me here again stress what was said earlier about assertibility. If one holds that vague expressions have no, or only trivial, semantic values, then one will want to draw a distinction between vague sentences that are properly assertible and those that are not. But if one succeeds in drawing such a distinction, can not the theory of assertibility one then can construct function equally well as an account of truth-conditions?

In his (1975), Michael Dummett says that the use of vague expressions is 'intrinsically inconsistent' and that there can be no 'coherent logic' of vague expressions. This is in an otherwise very careful and clear article, but Dummett does not elaborate much. And the slogans Dummett uses are not very clear. One can easily come up with uncharitable ways of reading Dummett. Start with the talk of 'no coherent logic'. What does this mean? That there is a logic of vague expressions, but it is incoherent? If so, what does this mean? A certain kind of theorist could propose that a paraconsistent logic is appropriate for a vague language, but that can hardly be what Dummett has in mind. Or that there is no logic of vague expressions? But what does that mean, in light of the fact that some arguments involving vague expressions are clearly valid and some are clearly non-valid. As for 'intrinsically inconsistent', what does this mean? Can I not use a vague expression, and with its customary meaning, but apply it only to some paradigm positive cases, disapply it only to some paradigm negative cases, and maintain a studious silence when it comes to everything in between? If so, the use I make of the expression is recognizably consistent.

This is not to say that one cannot interpret Dummett more charitably. The talk of the use of vague expressions being intrinsically inconsistent can be interpreted to mean that someone using a vague expression with its customary meaning takes on incoherent obligations or has incoherent dispositions. The talk of there being no coherent logic of vagueness can be interpreted to mean that there can be no logic of vagueness that respects the meaning-constitutive principles of all the expressions of our language. In other words, Dummett can be interpreted in such a way that what he says is fully consonant with what I want to say.

\section{REFERENCES}

Braun, David and Theodore Sider 2007. 'Vague, So Untrue', Noûs 41: 133-56.

Campbell, Richmond 1974. 'The Sorites Paradox', Philosophical Studies 26: 175-91.

Cherniak, Christopher 1984. 'Computational Complexity and the Universal Acceptance of Logic', Journal of Philosophy 81: 739-58.

Dummett, Michael 1975. 'Wang's Paradox"', Synthese 30: 201-32.

Dietz, Richard and Sebastiano Moruzzi (eds.) 2010 Cuts and Clouds. Oxford University Press. 
Eklund, Matti 2002. 'Inconsistent Languages', Philosophy and Phenomenological Research 64: 251-75.

Eklund, Matti 2005. 'What Vagueness Consists In', Philosophical Studies 125: 27-60.

Eklund, Matti 2007. 'Meaning-Constitutivity', Inquiry 50: 559-74.

Eklund, Matti 2010a. 'Review of Timothy Williamson, The Philosophy of Philosophy', Australasian Journal of Philosophy 88: 752-4.

Eklund, Matti 2010b. 'Vagueness and Second-Level Indeterminacy', in Dietz and Moruzzi (eds.).

Eklund, Matti 2013. 'Metaphysical Vagueness and Metaphysical Indeterminacy', Metaphysica 14: 165-79.

Eklund, Matti forthcoming. Choosing Normative Concepts. Oxford University Press.

Fara, Delia Graff 2000. 'Shifting sands: an interest-relative theory of vagueness', Philosophical Topics 28: 45-81. Published under the name Delia Graff.

Field, Hartry 1973. 'Theory Change and the Indeterminacy of Reference', Journal of Philosophy 70: 462-81.

Fine, Kit 1975. 'Vagueness, Truth, and Logic', Synthese 30: 265-300.

Heller, Mark 1990. The Ontology of Physical Objects. Cambridge University Press.

Hodes, Harold 1984. 'Logicism and the Ontological Commitments of Arithmetic', Journal of Philosophy 81: 123-49.

Horgan, Terence 1994. 'Robust Vagueness and the Forced-March Sorites Paradox', Philosophical Perspectives 8: 159-88.

Kamp, Hans 1981. 'The Paradox of the Heap', in Uwe Mönnich (ed.), Aspects of Philosophical Logic (Dordrecht: D. Reidel), pp. 225-77.

Keefe, Rosanna 2000. Theories of Vagueness. Cambridge University Press.

Lewis, David 1970. 'How to Define Theoretical Terms', Journal of Philosophy, 67: 42746.

Lewis, David 1972. 'Psychophysical and Theoretical Identifications', Australasian Journal of Philosophy 50: 249-58.

Lewis, David 1983. 'New Work for a Theory of Universals', Australasian Journal of Philosophy 61: 343-77.

Lewis, David 1984. 'Putnam's Paradox', Australasian Journal of Philosophy 62: 221-36.

Lewis, David 1997. 'Naming the Colours', Australasian Journal of Philosophy 75: 32542.

Ludwig, Kirk and Greg Ray 2002. 'Vagueness and the Sorites Paradox', Philosophical Perspectives 16: 419-61.

MacFarlane, John 2016. 'Vagueness as Indecision', Proceedings of the Aristotelian Society, Suppl. Vol. 90: 255-83.

Merricks, Trenton 2001. 'Varieties of Vagueness', Philosophy and Phenomenological 
Research 62: 145-57.

Raffman, Diana 2014. Unruly Words. Oxford University Press.

Scharp, Kevin 2013. Replacing Truth, Oxford University Press.

Schwarz, Wolfgang 2014. 'Against Magnetism', Australasian Journal of Philosophy 92: 17-36.

Shapiro, Stewart 2006. Vagueness in Context. Oxford University Press.

Smith, Nick 2008. Vagueness and Degrees of Truth. Oxford University Press.

Soames, Scott 1999. Understanding Truth. Oxford University Press.

Taylor, David and Alexis Burgess 2015. 'What in the World is Semantic Indeterminacy?', Analytic Philosophy 56: 298-317.

Unger, Peter 1979a. 'Why There are No People', Midwest Studies in Philosophy 5: 177222.

Unger, Peter 1979b. 'There are No Ordinary Things', Synthese 4: 117-54.

Unger, Peter 1979c. 'I Do Not Exist', in Graham Macdonald (ed.), Perception and Identity (Ithaca: Cornell University Press), pp. 177-222.

Unger, Peter 1980. 'Scepticism and Nihilism', Noûs 14: 517-45.

Weatherson, Brian 2010. 'Vagueness as Indeterminacy', in Dietz and Moruzzi (eds.).

Wheeler, Samuel C. III 1975. 'Reference and Vagueness', Synthese 30: 367-79.

Wheeler, Samuel C. III 1979. 'On That Which Is Not', Synthese 41: 155-94.

Williamson, Timothy 2007. The Philosophy of Philosophy, Blackwell, Oxford.

Williamson, Timothy: 2009. 'Reference, Inference, and the Semantics of Pejoratives', in Joseph Almog and Paolo Leonardi (eds.), The Philosophy of David Kaplan (Oxford University Press).

Wright, Crispin 1975. 'On the Coherence of Vague Predicates', Synthese 30: 325-65.

Wright, Crispin 2004. 'Intuition, Entitlement, and the Epistemology of Logical Laws', Dialectica 58: 155-75.

Zardini, Elia 2008. 'A Model for Tolerance', Studia Logica 90: 337-68. 